\title{
Metastatic Hepatic Epitheloid Hemangioendothelioma in a Young Male: A Rare Presentation
}

\author{
Tanweerul Huda Mohammad Masoom Parwez Bharati Pandya \\ Department of General Surgery, AlIMS, Bhopal, MP, India
}

\begin{abstract}
Keywords
Hemangioendothelioma $\cdot$ Epithelioid

hemangioendothelioma $\cdot$ Hepatic epithelioid

hemangioendothelioma $\cdot$ Vascular tumor $\cdot$ Rare tumor
\end{abstract}

\begin{abstract}
Hepatic EHE (epithelioid hemangioendothelioma) is an uncommon entity of vascular origin and a low-grade malignant tumor. Primary hepatic EHE is rare. These tumors can be multifocal at presentation like in the soft tissues, bones, brain, liver, and small intestine. First described by Weiss and Enzinger in 1982 as a malignant vascular neoplasm with indolent behavior. We report the case of a 23-year-old male, known case of chronic liver disease, who presented with incisional hernia following exploratory laparotomy 8 months back, performed for intestinal obstruction. Contrast-enhanced computed tomography abdomen revealed an incisional hernia with a large defect along with multiple lesions in the liver (suspected metastases) and peritoneal deposits and a few discrete lung nodules. Some areas of interloop collections were also noted. Biopsies were taken from the liver which revealed benign cirrhotic lesion. Relevant to the findings, multiple biopsies were performed and fluid was sent for evaluation. The liver biopsy came out positive for borderline vascular malignancy (epithelioid hemangioendothelioma). This was confirmed with the immunohistochemistry report. Epithelioid hemangioendothelioma occurs mostly in
\end{abstract}

karger@karger.com www.karger.com/gat

Karger $\stackrel{\text { ' }}{5}$

BOPEN ACCESS
(C) 2021 The Author(s)

Published by S. Karger AG, Basel

This article is licensed under the Creative Commons AttributionNonCommercial-NoDerivatives 4.0 International License (CC BYNC-ND) (http://www.karger.com/Services/OpenAccessLicense) Usage and distribution for commercial purposes as well as any distribution of modified material requires written permission. soft tissues of extremity and lungs. The involvement of the liver may be seen as metastasis or rarely as a primary tumor. The incidence of primary malignant hepatic hemangioendothelioma is about $0.1 / 100,000$; the mean age at the time of diagnosis is 41.7 years, and male:female ratio is 2:3. Liver transplantation, hepatectomy, chemoembolization, radiotherapy, and chemotherapeutic agents are reported treatment regimens. Malignant EHE of liver presents as multiple hepatic nodules. Being locally aggressive, it can invade the peritoneum, gut, and lungs. Orthoptic liver transplantation appears to be the only remedy because of the multifocal nature of the disease. Partial hepatectomy is possible for localized tumors.

(C) 2021 The Author(s) Published by S. Karger AG, Basel

\section{Introduction}

Malignant epithelioid hemangioendothelioma is a rare tumor of vascular origin that may present at multiple locations in the body. It was first described by Weiss and Enzinger in 1982 as an indolent malignant vascular neoplasm. Hepatic involvement is more of a rarer presentation. Only 200 cases are reported in the current literature. WHO classification 2002 describes EHE as locally aggressive tumors with metastatic potential. The biological behavior lies between benign hemangioma and malignant 


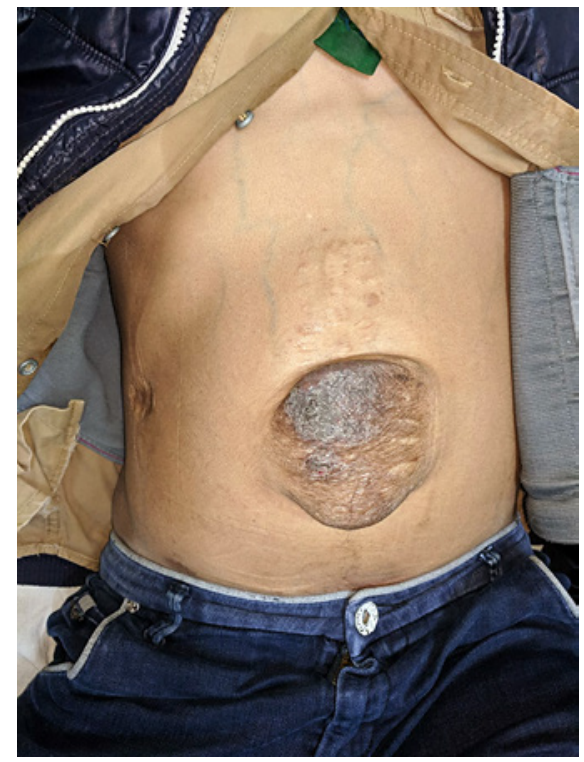

Fig. 1. Abdomen showing an incisional hernia over the infraumbilical region of the midline scar with overlying, hyperpigmented, and thinned out skin along with dilated veins over the abdomen.

angiosarcoma [1]. There are different risk factors associated with the development of hepatic EHE, like oral contraceptives, vinyl chloride, asbestos, thorotrast, hepatic trauma, and alcohol consumption [2]. Epithelioid hemangioendothelioma can involve the lungs, mediastinum, thyroid, peritoneum, lymph nodes, bone, palate, and liver [2].

We present the case of a 23 -year-old male with a history of abdominal swelling post laparotomy, with peritoneal and lung deposits. Excised tissue on histopathology suggested the diagnosis.

\section{Case Report}

A 23-year-old male patient presented with incisional hernia over the midline scar of a previous laparotomy incision done 8 months back for perforation peritonitis. He was a nonalcoholic, nonsmoker with no history of any other substance abuse. He was a diagnosed case of chronic liver disease developed after hepatitis over the past 5 years. Bowel and bladder habits were normal with no significant loss of appetite and weight.

Local examination of the abdomen revealed a $7 \times 7 \mathrm{~cm}$ defect with incisional hernia over the infraumbilical region of the midline scar with overlying, hyperpigmented, and thinned out skin (Fig. 1). Dilated veins were present over the abdomen surrounding the umbilicus. No visible peristalsis was noted. Free fluid was present.

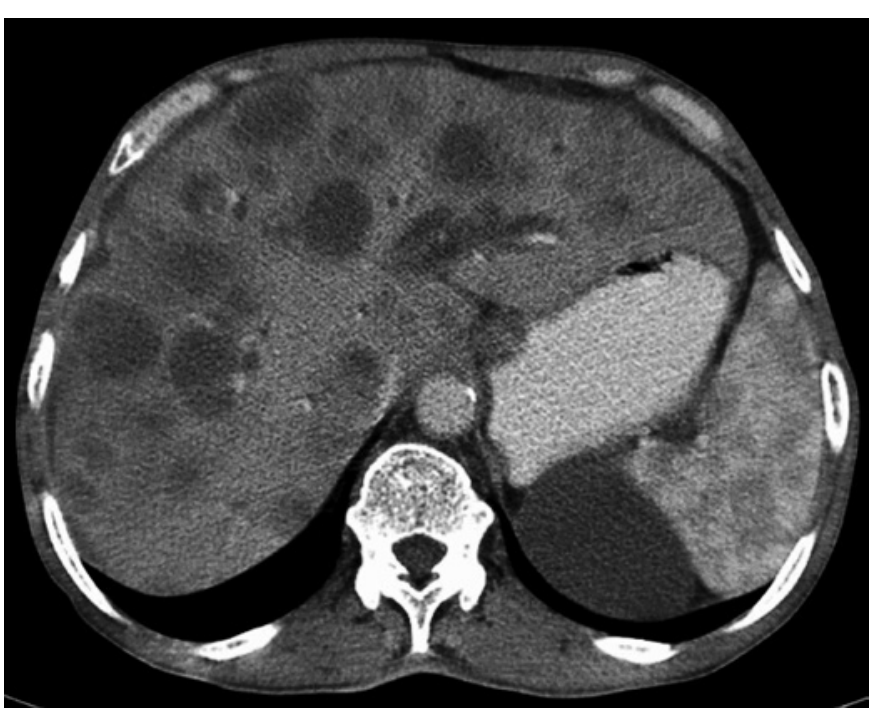

Fig. 2. CECT abdomen showing hepatomegaly with architectural distortion of the liver with multiple discrete and confluent lesions in the liver parenchyma showing peripheral enhancement in the arterial phase with central hypo-attenuating non-enhancing core. CECT, contrast-enhanced computed tomography.

External genitalia were normal, and digital rectal examination was unremarkable. A discrete, single, nontender, mobile, lymph node of $1 \times 1 \mathrm{~cm}$ size and firm in consistency was noted in the left supraclavicular region. A single discrete sub-centimetric lymph node was palpated in the left axillary region (central group).

Routine hematological investigations were within the reference range; liver function test was deranged with abnormally elevated alkaline phosphatase values. The coagulation profile was deranged with elevated prothrombin time, partial thromboplastin time, and international normalized ratio values. Viral markers were negative. Contrast-enhanced computed tomography abdomen revealed hepatomegaly with architectural distortion of the liver with multiple discrete and confluent lesions in the liver parenchyma showing peripheral enhancement in the arterial phase with central hypo-attenuating non-enhancing core, s/o neoplastic process. Multiple pockets of loculated ascites with internal septations along with multiple peritoneal deposits in the right paracolic gutter were found. A defect of $6.9 \mathrm{~cm}$ was noted in the midline in the anterior abdominal wall in the infraumbilical region with herniating omental fat and bowel loops (Fig. 2).

Exploration was done with midline laparotomy incision, the hernia was reduced, and anatomical repair approximating the linea alba was done. Thick-walled collection near the liver was visualized, and excised cyst wall was sent for histopathology; cyst fluid was aspirated and sent for routine biochemical investigation and cytology. The liver was stony hard, shrunken, and had a finely nodular surface with a deeply embedded porcelain gall bladder (Fig. 3). Multiple nodules were present and were hard and smooth within the omentum, over entire visceral as well as the parietal peritoneal surface (Fig. 4, 5). There were discrete pockets of chylous and se- 
3

Fig. 3. Picture showing shrunken liver with finely nodular surface along with a deeply embedded porcelain gall bladder.

Fig. 4. Picture showing multiple hard and smooth nodules within the omentum.

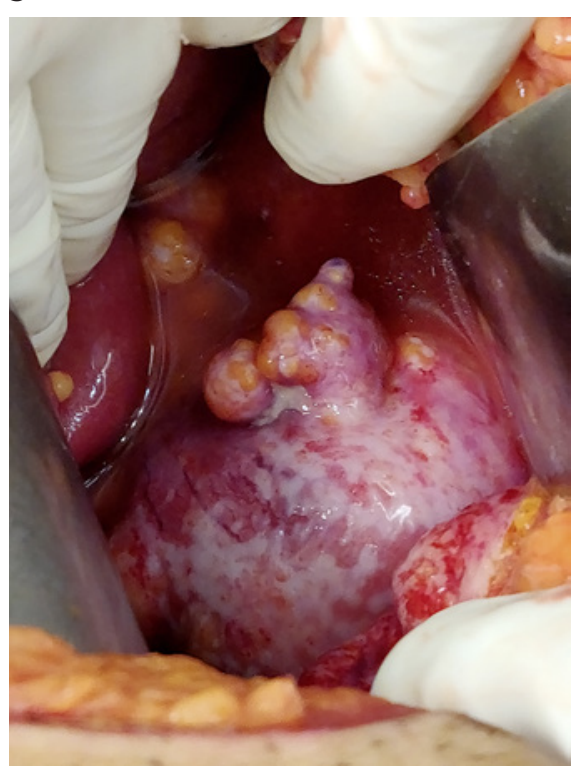

4

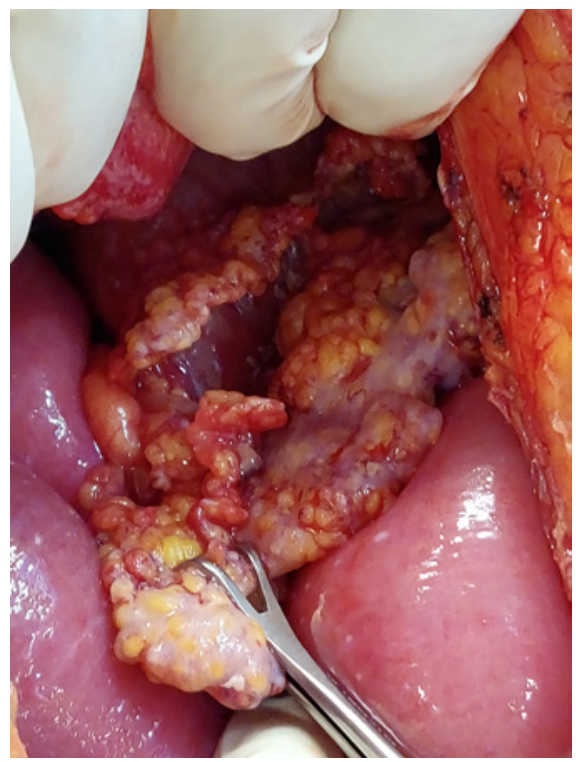

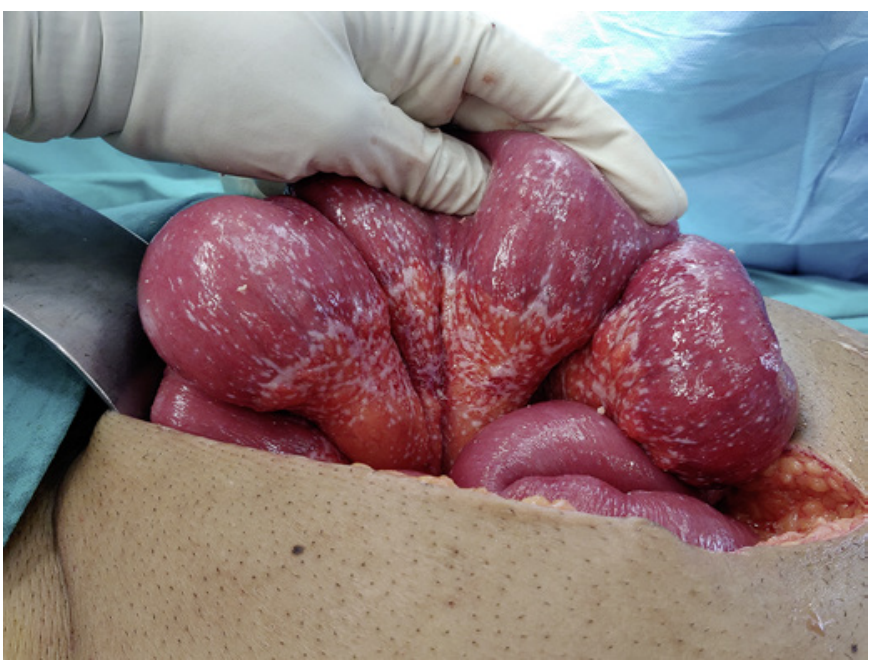

Fig. 5. Picture showing multiple nodules within the bowel mesentery.

rous collections with flimsy membranes containing them in the subhepatic, omental folds, peri-splenic region, and interloop areas. Gross portal system collaterals were noted. The spleen showed only a mild enlargement. Large nodules, possibly lymph nodes, were present in the portal region, subhepatic and peripancreatic region. The pancreas was normal. Peritoneal fluid was also sampled and sent for cytology. The liver tissue was biopsied and sent for histopathology. Enlarged abdominal groups of lymph nodes were also sampled and sent for histopathology. The postoperative period required meticulous care with protein supplementation, fluid-electrolyte balance, and respiratory rehabilitation, in view of subsequently developed ascites in the early postoperative period which responded well to medical management

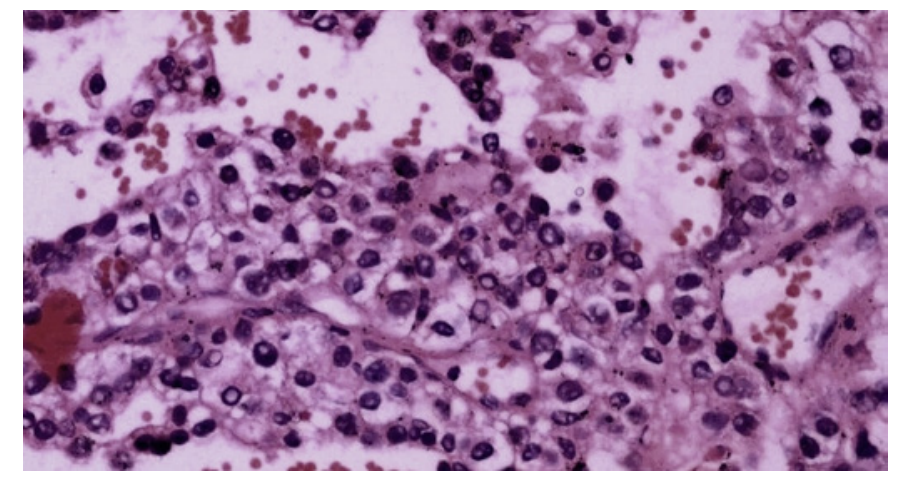

Fig. 6. Photomicrograph of an H\&E-stained section s/o borderline vascular malignancy, that is, epithelioid hemangioendothelioma.

\section{Histopathology Report}

Liver biopsy was positive for malignancy, s/o borderline vascular malignancy (epithelioid hemangioendothelioma) (Fig. 6). Abdominal lymph nodes showed metastatic tumor deposits (6/7). The excised cyst wall had peritoneal fibrosis with vascular proliferation. The skin over incisional hernia had cutaneous manifestations of portal hypertension in the form of dermal anastomosing channels. Cyst fluid was consistent with chylous fluid with raised triglyceride values $(259.34 \mathrm{mg} / \mathrm{dL})$. Peritoneal fluid showed lymphocyte predominance and singly scattered reactive mesothelial cells in a proteinaceous background. Stain for AFB was negative. Immunohistochemistry (IHC) showed positive for CD31, CD34, and cytokeratin. The patient was started on sorafenib in consultation with a medical oncologist.

In the follow-up for the past 6 months, the patient appeared well with no biochemical or hematological changes. No evidence of progression of disease in the form of deterioration in his health was noted.
Huda/Parwez/Pandya 


\section{Discussion}

Epithelioid hemangioendothelioma has a heterogeneous presentation as it represents $<1 \%$ of the vascular tumors. It is often misdiagnosed, leading to poor prognosis. Initially, it was named as an intravascular bronchioalveolar tumor as it was believed to be an aggressive form of bronchoalveolar cell carcinoma invading adjacent blood vessels and small airways. The term hemangioendothelioma was coined by Weiss and Enzinger in 1982 to describe a vascular tumor showing features between hemangioma and angiosarcoma.

The hepatic variant of hemangioendothelioma is relatively rare. Only 200 odd cases are reported in the current literature. The incidence of primary malignant hepatic hemangioendothelioma is $>0.1 / 100,000$; the mean age at the time of diagnosis is 41.7 years and the male:female ratio is 2:3.4. The etiology is still a dilemma. At the molecular level, different angiogenic stimulators may act as promoters of endothelial cell proliferation [3]. Recently, it has been reported that monocyte chemoattractant protein-1 is required for EHE proliferation and might promote the development of these lesions by stimulating the angiogenic behavior of endothelial cells [4]. A new causal relationship has been established between chronic Bartonella infection and this rare tumor [5].

Presentation of the disease is essentially nonspecific and the patients can have weight loss and right upper quadrant pain. In rare cases, jaundice and hepatic dysfunction may be noted due to replacement of liver parenchyma by the tumor [2].

Most nodules are hypoechoic on USG. On contrastenhanced computed tomography, these lesions appear as solid, nonhomogeneous, hypodense nodules with a lowdensity border and center. MRI usually shows a nonhomogeneous hypointense mass on T1-weighted images and hyperintense on T2-weighted images. On PET scan, hepatic EHE shows moderate to intense FDG uptake [6]. Radiologically, 2 distinct types of hepatic EHE with different stages have been described: the nodular type in the early stage of hepatic EHE and the diffuse type in advanced disease due to an increase in size and coalescence of the lesions often associated with hepatic vascular invasion [2].

The diagnosis is confirmed by histopathology. It includes an invasive growth pattern with the preservation of liver acinar composition and portal tracts. The tumor consists of epithelioid or dendritic cells, which are characterized by large eosinophilic cytoplasm in a fibromyxoid stroma [7]. Two striking histological features of hepatic EHE have been described: the presence of charac-

Metastatic Hepatic Epitheloid

Hemangioendothelioma teristic dendritic and/or epithelioid cells with evidence of vascular differentiation and intracytoplasmic lumina containing red blood cells, and abnormal stroma, which varies from myxomatous to densely fibrotic [2].

On IHC, the vascular structure is discerned with positive staining for endothelial markers. Factor VIII-related antigen is positive in almost $100 \%$ of cases, but the degree of staining between cells within a lesion can be highly variable. CD34 and CD31 are positive in 94 and $86 \%$ of cases, respectively. Other IHC stains that can be positive include factor XIIIa, vimentin, and type IV collagen. Tumors occasionally stain positive for cytokeratin markers and/or smooth muscle actin [8]. Positive podoplanin (D2-40) staining can help differentiate between hepatic EHE and other angiomatous lesions $[9,10]$. The most commonly identified genetic abnormality in EHE is CAMTA1-WWTR1 fusion product, with YAP1-TFE3 fusions also reported [8].

The tumor is heterogeneous in nature, and no specific treatment guidelines are mentioned in the literature. The management options for hepatic EHE include liver resection, liver transplantation, chemotherapy, radiotherapy, immunotherapy, or surveillance alone [11, 12]. Liver transplantation is an accepted treatment option for hepatic EHE and has the best outcome [2]. Multicentric tumors limit the scope of transplantation.

Different chemotherapy regimens have been suggested for metastatic hepatic EHE but there is no general consensus for a standard regimen. In the past, chemotherapy agents employed have been doxorubicin, vincristine, interferon alpha, 5-FU, thalidomide, and monoclonal antibodies against vascular endothelial growth factor $[8,13]$. One study described a 33-year-old patient treated with a combination of doxorubicin, vincristine, and 5-fluorouracil that had symptom-free survival of 10 years; another reported a patient with spleen and peritoneum involvement in whom endemic regression was observed after 3 cycles of doxorubicin, and another patient was administered an epirubicin and dacarbazine combination, but the metastases were not controlled [14, 15]. Thalidomide, due to its anti-angiogenic properties, has shown a significant effect in the treatment [16]. Furthermore, other drugs used include anti-VEGF monoclonal antibodies, that is, bevacizumab and ranibizumab, anti-VEGF aptamer, that is, pegaptanib, and inhibitor of VEGF receptor, that is, sunitinib $[17,18]$. Sangro et al. reported the treatment of a patient with metastatic hepatic EHE with sorafenib which is an inhibitor of tyrosine-kinase associated with VEGFR-2, VEGFR-3, and PDGF- $\beta$. It helped in long-term tumor stabilization in a patient with hepatic 
EHE along with lung and liver metastases [19]. Another study described the cyclophosphamide-based metronomic chemotherapy regimen which consisted of daily continuous administration of cyclophosphamide at low concentration. At this low concentration, immunological and anti-angiogenic functions were seen [20].

This case had diffuse peritoneal and pulmonary deposits with porta-hepatic and abdominal lymphadenopathy suggestive of aggressive disease. Thus, a liver transplant was ruled out due to multicentric nature. We dealt with the hernia component of the patient and started him on sorafenib in consultation with a medical oncologist.

\section{Conclusion}

Malignant EHE of the liver presents as multiple hepatic nodules. Being locally aggressive, it can invade the peritoneum, gut, and lungs. Orthoptic liver transplantation appears to be the only remedy because of the multifocal nature of the disease. Partial hepatectomy is possible for localized tumors. Whereas for metastatic tumors, chemotherapy and radiotherapy appear to be the only option available for this rare entity. Immunotherapy options also can be explored.

\section{Statement of Ethics}

Informed and written consent has been taken from the patient for the contents of the manuscript to be published including images.

\section{Conflict of Interest Statement}

The authors have no conflicts of interest to declare.

\section{Funding Sources}

The authors did not receive any funding.

\section{Author Contributions}

Tanweerul Huda contributed substantially to the concept, design, drafting the work, and final approval of the version to be published. Mohammad Masoom Parwez and Bharati Pandya contributed substantially in acquisition, interpretation, critically analyzing the data, and drafting the work.

\section{References}

1 Treska V, Daum O, Svajdler M, Liska V, Ferda J, Baxa J. Hepatic epithelioid hemangioendothelioma: a rare tumor and diagnostic dilemma. In Vivo. 2017;31(4):763-7.

2 Redaelli D, Guraya SS. Primary hemangioendothelioma of liver: report of a case and review of literature. J Taibah Univ Med Sci. 2015;10(2):243-7.

3 Weldon-Linne CM, Victor TA, Christ ML, Fry WA. Angiogenic nature of the intravascular bronchioloalveolar tumor of the lung: an electron microscopic study. Arch Pathol. 1981;105:174-9.

4 Gordillo GM, Onat D, Stockinger M, Roy S, Atalay M, Beck FM, et al. A key angiogenic role of monocyte chemoattractant protein-1 in hemangioendothelioma proliferation. Am J Physiol Cell Physiol. 2004;287(4):C866-73.

5 Mascarelli PE, Iredell JR, Maggi RG, Weinberg G, Breitschwerdt EB. Bartonella species bacteremia in two patients with epithelioid hemangioendothelioma. J Clin Microbiol. 2011;49(11):4006-12.

6 Choi KH, Moon WS. Epithelioid hemangioendothelioma of the liver. Clin Mol Hepatol. 2013;19(3):315.

7 Thin LW, Wong DD, De Boer BW, Ferguson JM, Adams L, Macquillan G, et al. Hepatic epithelioid haemangioendothelioma: chal- lenges in diagnosis and management. Intern Med J. 2010;40(10):710-5.

8 Studer LL, Selby DM. Hepatic epithelioid hemangioendothelioma. Arch Pathol Lab Med. 2018;142(2):263-7.

9 Fujii T, Zen Y, Sato Y, Sasaki M, Enomae M, Minato $\mathrm{H}$, et al. Podoplanin is a useful diagnostic marker for epithelioid hemangioendothelioma of the liver. Mod Pathol. 2008;21(2): 125-30.

10 Makhlouf HR, Ishak KG, Goodman ZD. Epithelioid hemangioendothelioma of the liver: a clinicopathologic study of 137 cases. Cancer. 1999;85(3):562-82.

11 Sahai P, Joshi P, Sharma ND, Haresh PK, Chandrashekhara HS, Julka KP, et al. Malignant epithelioid hemangioendothelioma of liver. Transl Gastrointest Cancer. 2012;1(3):272-6.

12 Mehrabi A, Kashfi A, Fonouni H, Schemmer P, Schmied BM, Hallscheidt P, et al. Primary malignant hepatic epithelioid hemangioendothelioma: a comprehensive review of the literature with emphasis on the surgical therapy. Cancer. 2006;107(9):2108-21.

13 Sanduzzi-Zamparelli M, Rimola J, Montironi C, Nunes V, Alves VAF, Sapena V, et al. Hepatic epithelioid hemangioendothelioma: an international multicenter study. Dig Liver Dis. 2020;52(9):1041-6.
14 Idilman R, Dokmeci A, Beyler AR, Bastemir M Ormeci N, Aras N, et al. Successful medical treatment of an epithelioid hemangioendothelioma of liver. Oncology. 1997;54(2):171-5.

15 Morris JE, Malatjalian DA, Bodurthra A, Smith $\mathrm{M}$, Koller O, Bhan J, et al. Epitheloid hemangioendothelioma of the liver: a report of two cases. Can J Gastroenterol. 1993;7:530-4.

16 Soape MP, Verma R, Payne JD, Wachtel M, Hardwicke F, Cobos E. Treatment of hepatic epithelioid hemangioendothelioma: finding uses for thalidomide in a new era of medicine. Case Rep Gastrointest Med. 2015;2015: 326795-4.

17 Breen EC. VEGF in biological control. J Cell Biochem. 2007;102(6):1358-67.

18 Sardaro A, Bardoscia L, Petruzzelli MF, Portaluri M. Epithelioid hemangioendothelioma: an overview and update on a rare vascular tumor. Oncol Rev. 2014;8(2):259-91.

19 Sangro B, Iñarrairaegui M, Fernández-Ros N Malignant epithelioid hemangioendothelioma of the liver successfully treated with sorafenib. Rare Tumors. 2012;4(2):e34-9.

20 Lakkis Z, Kim S, Delabrousse E, Jary M, Nguyen T, Mantion G, et al. Metronomic cyclophosphamide: an alternative treatment for hepatic epithelioid hemangioendothelioma. J Hepatol. 2013;58(6):1254-7. 